\title{
Aumento del daño en el ADN y estrés oxidativo en espermatozoides de pacientes con oligozoospermia idiopática y antecedentes de criptorquidismo
}

\author{
Rosita Smith G ${ }^{1 a}$, Heidy Kaune G ${ }^{2 a}$, Daniela Parodi $\mathrm{Ch}^{1 \mathrm{a}}$, \\ Marcia Madariaga A ${ }^{1 c}$, Ignacio Morales $D^{3}$, Rafael Ríos $S^{4}$, \\ Andrea Castro $\mathbf{G}^{\mathbf{1 b}}$. \\ Extent of sperm DNA damage in \\ spermatozoa from men examined \\ for infertility. Relationship with \\ oxidative stress
}

Background: Cryptorchidism and oligozoospermia are clinical conditions closely associated with impaired fertility. Oxidative stress and related sperm DNA damage have been identified as significant causes of male infertility. Aim: To determine the extent of sperm nuclear DNA damage in patients affected with idiopathic oligozoospermia or undescended testes and to examine its relationship with oxidative stress. Patients and methods: We studied 20 patients with idiopathic oligozoospermia and 18 with undescended testes (who previously underwent orchiopexy) and 25 normozoospermic healthy controls. All subjects underwent semen analysis. Sperm DNA damage was evaluated by the sperm chromatin structure assay/flow cytometry (SCSA-FCM) and by the dUTP-biotin nick end labeling (TUNEL) assay. Levels of reactive oxygen species (ROS) and total antioxidant capacity (TAC) were assessed by a chemiluminescence assay. Results: DFI (percentage of sperm with denatured DNA) values and percentage of TUNEL positive cells were significantly greater in patients with oligozoospermia (DFI: $28.8 \pm 5.6$; TUNEL+. $26.9 \pm 3.0$ ) or cryptorchidism (DFI: $26.4 \pm 10.1$; TUNEL+. 29.1 \pm 3.9 ), compared with controls (DFI: $7.1 \pm 0.9$; TUNEL+. $14.2 \pm 1.2$ ). Similarly, both groups of patients had significantly higher ( $\mathrm{p} \varangle 0.01$ ) levels of ROS. TAC levels did not differ between control and patient groups, suggesting that the DNA damage occurs before spermiation. Conclusions: Sperm DNA damage is significantly increased in men with idiopathic oligozoospermia and in cryptorchid subjects. The finding of increased ROSlevels may indicate that seminal oxidative stress may be involved in the pathogenesis of sperm DNA damage in these patients (Rev Méd Chile 2007; 135: 279-86).

(Key words: Cryptorchidism; Oligospermia; Oxidative stress)

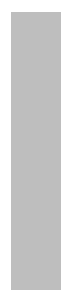

Recibido el 17 de abril, 2006. Aceptado el 28 de agosto, 2006.

Trabajo con financiamiento FONDECYT proyecto \#1011045.

${ }^{1}$ Instituto de Investigaciones Materno Infantil, Facultad de Medicina, Universidad de Chile. Hospital Clínico San Borja-Arriarán. ${ }^{2}$ Facultad de Ciencias de la Salud, Universidad Diego Portales. ${ }^{3}$ Servicio de Urología, Hospital Parroquial de San Bernardo. ${ }^{4}$ Servicio de Endocrinología Adulto, Facultad de Medicina, Universidad de Chile. Hospital Clínico San Borja- Amiarán.

aMagíster en Biología de la Reproducción

bMagíster en Biología Celular

'Tecnólogo Medico

Correspondencia a: Dra. Rosita Smith G. Santa Rosa 1234

Casilla 226-3 Santiago, Chile. Teléfono: 4248280. Fax: 56-2-

4247246. E mail: rsmith@med.uchile.cl 
$E^{1}$ daño en el ADN de los espermatozoides es considerado como una causa importante de infertilidad $\mathrm{y}$ ha despertado particular interés debido al riesgo que implica la transmisión de defectos genéticos a la descendencia, en especial, cuando se utilizan técnicas de reproducción asistida, donde no es posible realizar una selección espermática que permita excluir espermatozoides con daño genómico. Diversos estudios han demostrado que los espermatozoides humanos exhiben una alta tasa de daño en el ADN y que éste se acentúa en condiciones patológicas que conducen a infertilidad ${ }^{1-4}$. Dos situaciones andrológicas estrechamente asociadas a subfertilidad/infertilidad son la oligozoospermia y el criptorquidismo. La oligozoospermia se observa frecuentemente en pacientes que consultan por infertilidad y se caracteriza por una baja concentración de espermatozoides que va de moderada ( $<20$ millones de espermatozoides $/ \mathrm{ml}$ ) a severa $(<5 \text { millones de espermatozoides } / \mathrm{ml})^{5,6}$. Por otra parte, la criptorquidia constituye la anomalía congénita más frecuente observada en niños recién nacidos (4\%-5\%) y su incidencia parece haber aumentado en los últimos años ${ }^{7}$. Aun cuando estos pacientes comúnmente son intervenidos quirúrgicamente (orquidopexia), frecuentemente presentan oligo o azoospermia en la edad adulta ${ }^{7-9}$.

Numerosos autores han demostrado que el estrés oxidativo, juega un papel preponderante en la etiología de la infertilidad masculina ${ }^{10}$. La producción elevada de especies reactivas del oxígeno se asocia a una pérdida de la función espermática a través de mecanismos que incluyen lipoperoxidación de membranas, alteraciones del metabolismo, movilidad y capacidad fecundante de los espermatozoides $^{11,12}$. Además, se ha demostrado que el estrés oxidativo afecta directamente la integnidad genómica de los espermatozoides induciendo entrecruzamientos de la cromatina, oxidación de bases nitrogenada y rupturas de las hebras del $\mathrm{ADN}^{13}$.

Con el propósito de esclarecer los mecanismos fisiopatológicos que conducen a infertilidad en estos pacientes, el primer objetivo de este estudio fue evaluar la extensión del daño en el ADN nuclear de espermatozoides de pacientes que consultan por infertilidad y que presentan diagnóstico clínico de oligozoospermia idiopática 0 antecedentes de criptorquidismo. El segundo objetivo fue determinar la relación entre el daño en el ADN espermático y el estrés oxidativo seminal.

\section{PACIENTES Y MÉTODOS}

Pacientes. El grupo estudiado incluyó 35 pacientes que consultaron por infertilidad primaria, en 20 de ellos el diagnóstico fue de oligozoospermia idiopática y 18 presentaron antecedentes de criptorquidia. El grupo control estuvo constituido por 25 individuos sanos con características seminales de normozoospermia ${ }^{14}$. En todos los pacientes y controles se realizó una historia médica y un examen físico completo y se midieron las concentraciones plasmáticas de FSH, LH y testosterona. En cada sujeto se realizaron dos análisis seminales con un intervalo de una semana. Se excluyeron del estudio todos los sujetos que presentaron testículo retráctil, leucocitospermia, varicocele y exposición a calor, sustancias tóxicas, alcoholismo y tabaquismo crónico. Pacientes y controles dieron consentimiento escrito para participar en este estudio, el cual fue aprobado por el Comité de Etica de nuestra institución.

Análisis seminal. Se realizó siguiendo los criterios recomendados por la OMS ${ }^{14}$. Los parámetros seminales se consideraron normales cuando la concentración espermática fue $\geq 20 \times 10^{6} / \mathrm{ml}$, la movilidad progresiva $\geq 40 \%$, y el porcentaje de espermatozoides con morfología normal $\geq 30 \%$.

Determinación de parámetros asociados a estrés oxidativo: especies reactivas del oxígeno (EROs) y capacidad antioxidante del plasma seminal (CAT). La determinación de los niveles de EROs en las suspensiones de espermatozoides se realizó por quimioluminiscencia utilizando luminol (Sigma, USA ${ }^{15}$. Cada suspensión de espermatozoides fue incubada con luminol y la señal registrada en un luminómetro (Hidex, Finland). Las mediciones se registraron cada $20 \mathrm{~s}$ durante $5 \mathrm{~min}$. Los resultados expresados como cuentas/20 s/3x106 de espermatozoides fueron normalizados a Log $($ EROs +1$)$. El estado de los antioxidantes totales se determinó en plasma seminal utilizando un kit comercial (Total Antioxidant Status Assay Kit, Calbiochem). Las mediciones fueron realizadas siguiendo el protocolo descrito por Smith y cols ${ }^{16}$. Se registró la absorvancia en espectrofotómetro indicador de una condición de estrés oxidativo (Jenway 6405, UK). Los resultados fueron expresados como concentración de antioxidantes (mM). 
Determinación del daño en el ADN de los espermatozoides. Se evaluó tanto el daño resultante de un proceso de fragmentación, a través del ensayo de TUNEL, como las alteraciones de la cromatina, mediante el ensayo de estructura de la cromatina (SCSA). Estas metodologías no permiten evaluar otras causas de daño en el ADN, tales como, mutaciones y alteraciones cromosómicas numéricas y estructurales.

Ensayo de TUNEL Se utilizó el kit comercial «nsitu Cell Death Detection Kit, Fluorescein» (Roche, Mannheim, Alemania). Los frotis de las suspensiones de espermatozoides fueron fijados, permeabilizados e incubados en presencia de los nucleótidos marcados y de la enzima terminal deoxinucleotidil transferasa. Posteriormente, se realizó una contra-tinción con yoduro de propidio. Las placas fueron evaluadas en un microscopio de fluorescencia (Nikon). Para cada sujeto se observaron 500 espermatozoides. Los espermatozoides con ADN fragmentado presentaron fluorescencia verde, los espermatozoides normales mostraron fluorescencia roja. El coeficiente de variación interensayo fue $<7 \%$.

Ensayo de SCSA. Esta metodología permite monitorear la susceptibilidad de la cromatina a la denaturación ácida in situ ${ }^{17}$. Los espermatozoides fueron tratados con una solución detergente a $\mathrm{pH}$ bajo y posteriormente teñidos con naranja de acridina. El tratamiento denatura sólo el ADN de los espermatozoides que presentan alteraciones de la cromatina. Las células fueron analizadas en un citómetro de flujo (Becton Dickinson, San José, Ca). Para cada sujeto se realizó la adquisición de 50.000 eventos. La población de espermatozoides con cromatina normal presentó fluorescencia verde (hebra doble) y la anormal (hebra simple) fluorescencia roja. La extension del daño en el ADN se expresó como índice de fragmentación del ADN (DFI) que representa el porcentaje de espermatozoides con cromatina alterada. La variabilidad interensayo fue inferior a $4 \%$.

Estadística. El análisis de los datos se realizó con el programa GraphPadPrism ${ }^{\circledR}$ versión 3.0 (San Diego CA). Para comparar variables paramétricas se aplicó una prueba de ANOVA seguida del test de comparación múltiple de Tukeys. Las variables no paramétricas se determinaron mediante una prueba de t de Student o Mann-Whitney. Para correlacionar los distintos parámetros se utilizó el test de Spearman. El nivel de significancia se estableció en 0,05.

\section{Resultados}

Parámetros seminales y perfil hormonal. La edad y características seminales de los grupos estudiados se muestran en la Tabla 1. En ambos grupos de pacientes, a excepción del volumen seminal, los parámetros seminales presentaron valores significativamente inferiores a los del grupo control. En los pacientes con historia de criptorquidia, 5\% (1 de 18) presentó parámetros seminales normales y $17 \%$ presentó azoospermia. Estos últimos fueron eliminados del estudio. Ambos grupos de estudio fueron comparables en edad. La Tabla 2 muestra los niveles de FSH, $\mathrm{LH}$ y testosterona (promedio $\pm \mathrm{EE}$, rangos y valores de referencia) en controles y pacientes. Ambos grupos de pacientes presentaron valores promedio de $\mathrm{LH}$ y testosterona dentro del rango normal. Los niveles de FSH fueron significativamente superiores en los pacientes con antecedentes de criptorquidia. En estos pacientes, 50\% presentó niveles de FSH superior a $7 \mathrm{mUI} / \mathrm{ml}$.

Marcadores asociados a estrés oxidativo. En la Figura 1A se comparan los niveles de EROs en controles y pacientes. La producción de EROs en espermatozoides de pacientes con oligozoospermia $(4,4 \pm 0,3)$ y con historia de criptorquidia $(5,2 \pm 0,6)$ fue significativamente superior a la observada en el grupo de sujetos controles $(2,8 \pm 0,9)$.

La Figura 1B representa los valores promedios $( \pm$ $\mathrm{EE}$ ) y los rangos del estado de los antioxidantes en el plasma seminal de sujetos controles y pacientes. No se observaron diferencias significativas, en los valores promedios de este parámetro, entre el grupo control $(1,2 \pm 0,1 \mathrm{mM})$ y los grupos de pacientes con oligozoospermia $(1,2 \pm 0,1 \mathrm{mM})$ y antecedentes de criptorquidia $(1,3 \pm 0,3 \mathrm{mM})$. El $27 \%$ de los pacientes oligozoospérmicos y $46 \%$ de los con antecedentes de criptorquidia presentaron niveles de antioxidantes bajo el límite inferior del grupo control $(\varangle 0,9 \mathrm{mM})$.

Fragmentación del ADN determinada a través del ensayo de TUNEL El porcentaje promedio de 
Tabla 1. Edad y características seminales en sujetos controles y pacientes

\begin{tabular}{|lccc|}
\hline & Control & Oligozoospermia & Criptorquidia \\
\hline \multirow{2}{*}{ Edad } & $31,7 \pm 0,9$ & $31,7 \pm 1,9$ & $30,1 \pm 2,3$ \\
Volumen (ml) & $21-37$ & $22-48$ & $18-42$ \\
& $2,9 \pm 0,3$ & $2,8 \pm 0,2$ & $2,7 \pm 0,3$ \\
Concentración $(106 / \mathrm{ml})$ & $0,7-7,3$ & $1,4-4,6$ & $0,9-4,2$ \\
& $102,3 \pm 10,6$ & $7,7 \pm 1,3^{*}$ & $8,4 \pm 4^{*}$ \\
Recuento (106) & $31,4-215$ & $0,1-22,4$ & $0-53,2$ \\
& $254,6 \pm 35,1$ & $19,4 \pm 3^{*}$ & $28,7 \pm 15,2^{*}$ \\
Viabilidad (\%) & $56,8-920$ & $0,3-44,8$ & $0-212,8$ \\
& $92 \pm 0,8$ & $77,1 \pm 2,1^{*}$ & $76 \pm 5^{*}$ \\
Movilidad progresiva (\%) & $80-97$ & $60-90$ & $43-94$ \\
Morfología normal OMS (\%) & $77,7 \pm 1,7$ & $37,6 \pm 3,6^{*}$ & $37,5 \pm 7,9^{*}$ \\
& $59-89$ & $14-69$ & $0-72$ \\
& $56,7 \pm 2,4$ & $31,3 \pm 3,7^{*}$ & $33,2 \pm 5,4^{*}$ \\
\hline
\end{tabular}

Los valores representan promedios $\pm \mathrm{DE}$; números bajo los promedios corresponden a rangos. $* p<0,001$.

Tabla 2. N iveles de gonadotropinas y testosterona en sujetos controles y pacientes

\begin{tabular}{|lcccccc|}
\hline \multirow{2}{*}{ V de ref. adulto } & \multicolumn{2}{c}{ FSH } & \multicolumn{2}{c}{$\mathrm{LH}$} & \multicolumn{2}{c|}{ Testosterona } \\
& \multicolumn{2}{c}{$1-7 \mathrm{mUI} / \mathrm{ml}$} & \multicolumn{2}{c|}{$1-8 \mathrm{mUI} / \mathrm{ml}$} & \multicolumn{2}{c|}{$2-8 \mathrm{ng} / \mathrm{ml}$} \\
& $\mathrm{X} \pm \mathrm{EE}$ & Rangos & $\mathrm{X} \pm \mathrm{EE}$ & Rangos & X EE & Rangos \\
\hline Control & $2,1 \pm 0,2$ & $1,5-2,9$ & $2,9 \pm 0,8$ & $0,8-6,0$ & $4,5 \pm 0,6$ & $3-7,8$ \\
Oligozoospermia & $5,5 \pm 0,9^{*}$ & $2,2-11,5$ & $3,6 \pm 0,6$ & $2,1-8,5$ & $5,9 \pm 0,5$ & $3,2-9,3$ \\
Criptorquidia & $9,2 \pm 1,6^{* *}$ & $1,9-17$ & $5,2 \pm 0,8$ & $2,1-8,4$ & $5,9 \pm 0,6$ & $4,5-8,4$ \\
\hline
\end{tabular}

$* p<0,01 . \quad * * p<0,001$.

espermatozoides con fragmentación del ADN aumentó desde $14,2 \%$ en sujetos controles a $26,9 \%$ y $29,1 \%$ en pacientes con oligozoospermia idiopática e historia de criptorquidia, respectivamente (Figura 2A). Es interesante notar que $50 \%$ de los pacientes oligozoospérmicos y $100 \%$ de los con criptorquidismo presentaron valores elevados (media grupo control más 2DE) de fragmentación en el ADN.

Daño en el ADN espermático determinado a través de la estructura de la cromatina. En la Figura 2B se presentan los resultados obtenidos en el análisis de la cromatina espermática en controles y pacientes. El valor promedio de los DFI (porcentaje de espermatozoides con ADN denaturado) en el grupo control fue de 7,1\% con un límite inferior y superior de 1,5 y 17,4, respectivamente. Este valor fue significativamente superior en el grupo de pacientes con oligozoospermia idiopática $(28,8 \pm 5,6)$ e historia de criptorquidismo $(26,4 \pm 10,3)$. Al relacionar los niveles de EROs y el daño en la estructura de la cromatina (DFI) se observó una comelación positiva significativa entre ambos $(p<0,05)$ en el grupo de pacientes. En el grupo control la extensión del daño en el ADN no se correlacionó con la producción de EROs. 


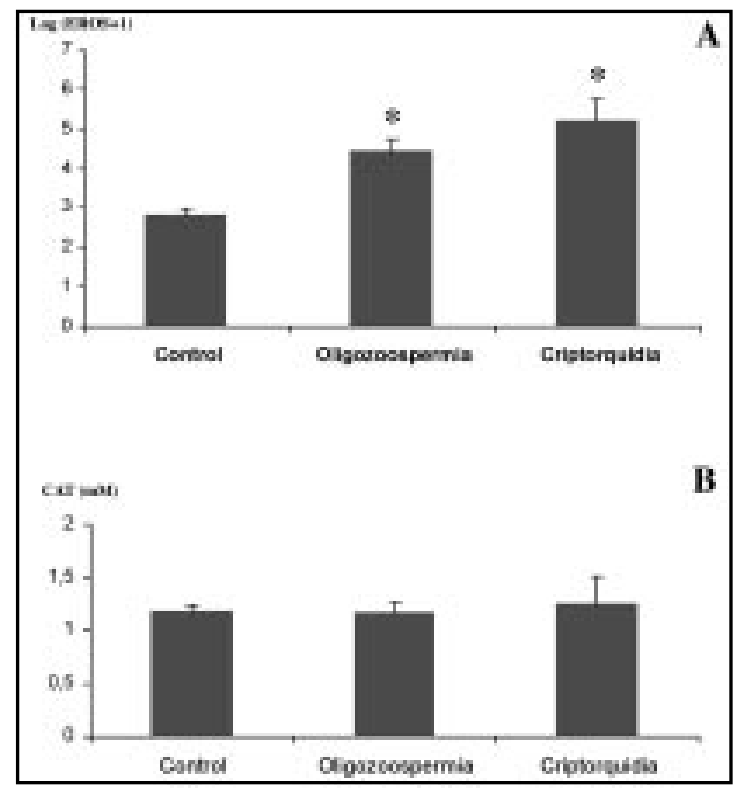

Figura 1. Comparación de los marcadores de estrés oxidativo en espermatozoides de sujetos controles y pacientes. A. Producción total de EROs. B. Capacidad antioxidante del plasma seminal. EROs: especies reactivas del oxígeno.

Los valores representan promedios \pm EE. ${ }^{*} p<0,001$.

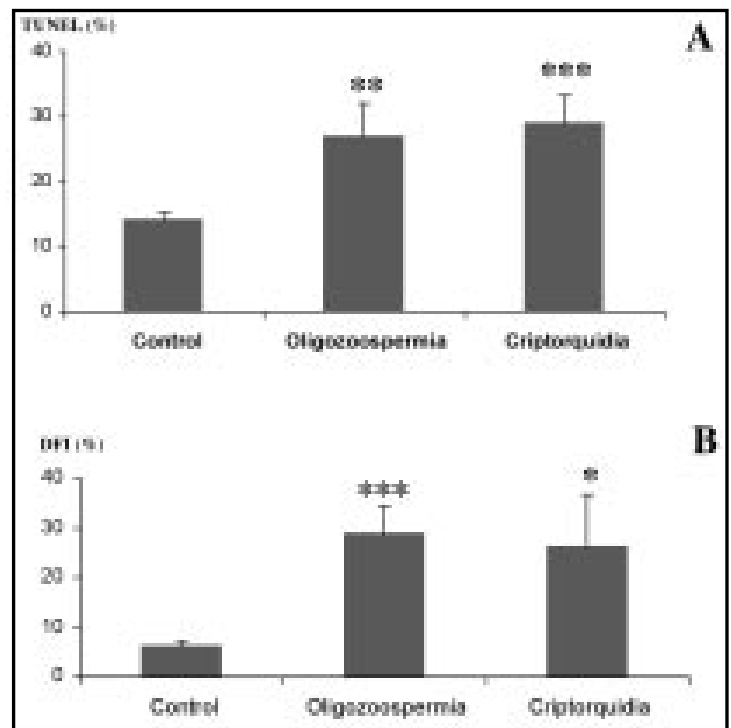

Figura 2. Determinación del daño en el ADN en sujetos controles y pacientes. A. Fragmentación del ADN evaluado a través del ensayo del TUNEL B. Alteraciones en la estructura de la cromatina (DFI) evaluada a través de SCSA. Los valores representan promedios $\pm \mathrm{EE}$. ${ }^{*} p<0,05$. $\quad$ **p $^{2}<0,01$. $\quad{ }^{* * *} p<0,001$.

\section{Discusión}

Los resultados de este estudio demostraron que el nivel de daño en el ADN de los espermatozoides eyaculados, se acentúa significativamente en las condiciones fisiopatológicas estudiadas. Los dos grupos de pacientes presentaron consistentemente una disminución de la calidad de la cromatina (SCSA) y un mayor porcentaje de espermatozoides con fragmentación del ADN (TUNEL). Además, nuestros resultados demostraron un aumento significativo en la producción de EROs en ambos grupos de pacientes, lo que sugiere que el estrés oxidativo seminal podría estar involucrado en la patogénesis del daño en el ADN espermático.

Se estima que $50 \%$ de las causas de infertilidad masculina son de origen idiopático. La etiología de algunas de estas causas podría estar relacionada con daño en el ADN de los espermatozoides. La oligozoospermia idiopática es comúnmente diagnosticada por exclusión de otras patologías, tales como, varicocele, criptorquidia y obstrucción parcial de la vía seminal. Existen numerosos hallazgos clínicos y de laboratorio que se asocian a oligozoospermia, éstos incluyen: la edad, la patología inflamatoria, autoinmune, disfunciones hormonales, ingesta de alcohol y factores genéticos e idiopáticos. Entre los factores idiopáticos, las alteraciones en el genoma de los gametos y en las mitocondrias, la presencia de tóxicos ambientales y alteraciones hormonales leves podrían constituir probables mecanismos que conduzcan a una desregulación de la homeostasis testicular.

Por otra parte, el criptorquidismo constituye una causa conocida de infertilidad masculina que afecta, aproximadamente, a 5\%-10\% de las parejas que consultan por infertilidad y representa además un factor de riesgo de cáncer testicular ${ }^{18,19}$. La exposición del testículo a la temperatura corporal resulta en una degeneración de las células germinales, por consiguiente, los pacientes que presentan testículos intrabdominales o intracanaliculares manifiestan una rápida disminución de la población de células germinales ${ }^{8,20,21}$. Aun cuando, la orquidopexia en etapa preescolar es reconocida como el tratamiento más adecuado en numerosos países, a menudo estos pacientes presentan alteraciones de la espermatogénesis y disminución del potencial fértil en el adulto. En la mayoría de los pacientes oligozoospérmicos y con 
historia de criptorquidismo, por sus características seminales, una opción terapéutica efectiva para lograr el embarazo es a través de los procedimientos de inseminación intrauterina y reproducción asistida $^{22}$.

En los pacientes con antecedentes de criptorquidismo, la extensión del daño en el ADN fue superior en dos desviaciones estándar por sobre la media del grupo control, en todos los sujetos estudiados. En estos pacientes, la orquidopexia fue realizada más tardíamente ( 3 a 20 años) que lo recomendado en la práctica clínica (hasta los 2 años) ${ }^{7,23}$. En general, existe consenso que la corrección quirúrgica a edad temprana contribuiría a disminuir el daño y mantener la función testicular en la adultez ${ }^{9,24}$. Sin embargo, es importante destacar que la etiología del criptorquidismo es multifactorial y se asocia no sólo a la permanencia del testículo en una localización extraescrotal, sino también, a varios otros factores que incluyen anomalías neurológicas, endocrinas y genéticas ${ }^{25}$.

En la actualidad no está claro si el daño en el ADN espermático es el resultado de un proceso generado a nivel testicular o si se origina de novo a nivel postesticular 0 ambos. Para explicar el origen del daño genómico, se han propuesto varios mecanismos, que incluyen: defectos en la compactación de la cromatina durante la espermiogénesis; aceleración del proceso de apoptosis en las células germinales testiculares, microdeleciones y alteraciones cromosómicas originadas durante la meiosis. Un ejemplo conocido de un factor intrínseco o extrínseco que es clave en la etiología de la infertilidad masculina es el estrés oxidativo. Un aumento significativo en la producción de EROs ha sido reportada en pacientes que consultan por infertilidad, que presentan parámetros seminales alterados y en condiciones patológicas que conducen a infertilidad ${ }^{26}$. En efecto, estudios previos han demostrado un aumento en las concentraciones de EROs en espermatozoides de pacientes infértiles con varicocele lo cual sugiere que la infertilidad en estos pacientes podría estar relacionada con el estrés oxidativo ${ }^{10,16}$. Nuestros resultados demuestran que los espermatozoides de pacientes con oligozoospermia idiopática y aquellos con antecedentes de criptorquidismo, producen niveles significativamente superiores de EROs, comparados con los del grupo control. Como otro indicador de estrés oxidativo evaluamos el estado de los antioxidantes no enzimáticos en el plasma seminal. Los niveles de antioxidantes no mostraron diferencias significativas entre controles y pacientes, lo que podría estar indicando que el daño en el ADN ocurriría antes de la espermiación, como resultado del efecto deletéreo de la patología sobre la función testicular. Es probable que en los grupos de pacientes estudiados, la exposición prolongada del epitelio seminífero a concentraciones suprafisiológicas de EROs podría conducir a una disminución de la integridad de la cromatina en las células germinales testiculares.

En este sentido, los parámetros indicadores de estrés oxidativo y de daño al ADN, porcentaje de espermatozoides con ADN fragmentado y DFI, constituyen marcadores moleculares que permiten identificar un grupo de pacientes que podrían beneficiarse de una terapia in vivo con antioxidantes para restablecer niveles adecuados de EROs.

La integridad del genoma paterno es esencial para asegurar la transmisión precisa y fidedigna de la información genética a la descendencia. Para determinar la importancia clínica (valor diagnóstico y pronóstico) del daño en el ADN espermático, es crítico conocer el impacto de éste sobre el desarrollo embrionario preimplantacional y posimplantacional. A este respecto, evidencias recientes sugieren que la fecundación de un ovocito con espermatozoides que exhiben niveles altos de fragmentación en el ADN, puede conducir a una pérdida embrionaria temprana, disminución de las tasas de embarazo ${ }^{27}$, morbilidad en la descendencia, incluido cáncer durante la niñez ${ }^{28}$, infertilidad en la adultez ${ }^{2,10,26}$, enfermedades genéticas causadas por mutaciones dominantes ${ }^{29}$ y enfermedades epigenéticas $^{30}$. En este contexto, varios estudios recientes han examinado la relación entre la fragmentación del $\mathrm{ADN}$, la calidad embrionaria, el clivaje y las tasas de embarazo tanto en la concepción natural ${ }^{31}$, como asistida ${ }^{32}$. En general, estos estudios demuestran una asociación negativa entre el porcentaje de espermatozoides con fragmentación del ADN y los resultados de la inseminación intrauterina, fecundación in vitro e inyección intracitoplasmática de espermatozoides ${ }^{33}$. Si bien, la evidencia disponible indica que el análisis del ADN espermático es una herramienta clínica valiosa en la evaluación del hombre infértil y, que existe una 
clara asociación entre los niveles de daño en el ADN y el potencial fértil del individuo; su valor predictivo tanto en la concepción natural como asistida, no ha sido claramente establecido ${ }^{34}$.

En síntesis, en este estudio hemos demostrado que los espermatozoides de pacientes con oligozoospermia idiopática y con antecedentes de criptorquidia, producen niveles elevados de EROs y un incremento significativo en la extensión del daño en el ADN. En estas situaciones andrológi-

\section{REFERENCIAS}

1. AitKen RJ, Koopman P, Lewis SE. Seeds of concem. Nature 2004; 432: 48-52.

2. Agarwal A, Said TM. Role of sperm chromatin abnormalities and DNA damage in male infertility. Hum Reprod Update 2003; 9: 331-45.

3. Saleh RA, Agarwal A, Sharma RK, Said TM, SikKa SC, THOMAs AJ JR. Evaluation of nuclear DNA damage in spermatozoa from infertile men with varicocele. Fertil Steril 2003; 80: 1431-6.

4. TESARIK J. Sperm nuclear DNA damage: update on the mechanism, diagnosis and treatment. Reprod Biomed Online 2006; 12: 715-21.

5. Aziz N, Agarwal A, Nallella KP, Thomas AJ Jr. Relationship between epidemiological features and aetiology of male infertility as diagnosed by a comprehensive infertility service provider. Reprod Biomed Online 2006; 12: 209-14.

6. Barri PN, Vendrell JM, Martínez F, Coroleu B, Aran B, VeIgA A. Influence of spermatogenic profile and meiotic abnormalities on reproductive outcome of infertile patients. Reprod Biomed Online 2005; 10: 735-9.

7. BaRTHOLD JS, González R. The epidemiology of congenital cryptorchidism, testicular ascent and orchiopexy. J Urol 2003; 170: 2396-401.

8. Rusnack SL, Wu HY, Huff DS, Snyder HM, $3^{\text {RD }}$, Carr MC, Bellah RD et al. Testis histopathology in boys with cryptorchidism correlates with future fertility potential. J Urol 2003; 169: 65962.

9. Caroppo E, Niederberger C, Elhanbly S, Schoor R, Ross L, D'Amato G. Effect of cryptorchidism and retractile testes on male factor infertility: a multi- cas, el estrés oxidativo podría constituir un mediador de daño testicular que, entre otros efectos, conduzca a una disminución de la integridad del $\mathrm{ADN}$ en las células germinales. La pérdida de la integridad genómica de los espermatozoides, cualquiera sea su origen, tiene profundas implicancias en el potencial fértil del individuo, particularmente si en estos pacientes se indica reproducción asistida como opción terapéutica para su infertilidad.

center, retrospective, chart review. Fertil Steril 2005; 83: 1581-4.

10. Agarwal A, Saleh RA, Bedaiwy MA. Role of reactive oxygen species in the pathophysiology of human reproduction. Fertil Steril 2003; 79: 829-43.

11. Moustafa MH, Sharma RK, Thornton J, Mascha E, Abdel-Hafez MA, Thomas AJ Jr et al. Relationship between ROS production, apoptosis and DNA denaturation in spermatozoa from patients examined for infertility. Hum Reprod 2004; 19: 129-38.

12. Wang $X$, Sharma RK, SikKa SC, Thomas AJ JR, Falcone T, Agarwal A. Oxidative stress is associated with increased apoptosis leading to spermatozoa DNA damage in patients with male factor infertility. Fertil Steril 2003; 80: 531-5.

13. SHen HM, ChiA SE, Ong CN. Evaluation of oxidative DNA damage in human sperm and its association with male infertility. J Androl 1999; 20: 718-23.

14. WHO. [Laboratory manual of the WHO for the examination of human semen and sperm-cervical mucus interaction]. Ann Ist Super Sanita 2001; 37: I-XII, 1-123.

15. Kobayashi H, Gil-Guzman E, Mahran AM, Rakesh, Nelson DR, Thomas AJ JR ET al. Quality control of reactive oxygen species measurement by luminol-dependent chemiluminescence assay. J Androl 2001; 22: 568-74.

16. Smith R, Kaune H, Parodi D, Madariaga M, Ríos R, MoRALES I ET AL. Increased sperm DNA damage in patients with varicocele: relationship with seminal oxidative stress. Hum Reprod 2006; 21: 98693.

17. Evenson DP, LaRson KL, Jost LK. Sperm chromatin structure assay: its clinical use for detecting 
sperm DNA fragmentation in male infertility and comparisons with other techniques. J Androl 2002; 23: 25-43.

18. Thonneau PF, Gandia P, Mieusset R. Cryptorchidism: incidence, risk factors, and potential role of environment; an update. J Androl 2003; 24: 15562.

19. HerRinton LJ, Zhao W, Husson G. Management of cryptorchism and risk of testicular cancer. Am J Epidemiol 2003; 157: 602-5.

20. Dunkel L, Taskinen S, Hovatta O, Tily JL, Wikstrom S. Germ cell apoptosis after treatment of cryptorchidism with human chorionic gonadotropin is associated with impaired reproductive function in the adult. J Clin Invest 1997; 100: 2341-6.

21. Han SW, Lee T, Kim JH, Chо SK, Сно NH, Han JY. Pathological difference between retractile and cryptorchid testes. J Urol 1999; 162: 878-80.

22. Raman JD, Schlegel PN. Testicular sperm extraction with intracytoplasmic sperm injection is successful for the treatment of nonobstructive azoospermia associated with cryptorchidism. J Urol 2003; 170: 1287-90.

23. WenZier DL, Bloom DA, Park JM. What is the rate of spontaneous testicular descent in infants with cryptorchidism? J Urol 2004; 171: 849-51.

24. Coughun MT, Bewnger MF, LeE PA. Age at unilateral orchiopexy: effect on hormone levels and sperm count in adulthood. J Urol 1999; 162: 9868; discussion 989.

25. Feng S, Cortessis VK, Hwang A, Hardy B, Кoh CJ, Bogatcheva NV, et al. Mutation analysis of INSL3 and GREAT/LGR8 genes in familial cryptorchidism. Urology 2004; 64: 1032-6.
26. AitKen RJ, BAKer MA, SAWyer D. Oxidative stress in the male germ line and its role in the aetiology of male infertility and genetic disease. Reprod Biomed Online 2003; 7: 65-70.

27. Henkel R, Hajimohammad M, Stalf T, Hoogendijk C, Mehnert C, MenkVeld R et al. Influence of deoxyribonucleic acid damage on fertilization and pregnancy. Fertil Steril 2004; 81: 965-72.

28. Pettersson A, Kaijser M, Richiardi L, Askung J, Еквом A, AкRE O. Women smoking and testicular cancer: one epidemic causing another? Int J Cancer 2004; 109: 941-4.

29. KuHNERT B, NiesCHIAG E. Reproductive functions of the ageing male. Hum Reprod Update 2004; 10: 327-39.

30. Valenzuela CY. [The risk of congenital malformations and genomic imprinting defects in assisted reproductive technologies and nuclear transfer cloning.]. Rev Méd Chile 2005; 133: 1075-80.

31. Spano M, Bonde JP, Hjolund HI, Kolstad HA, CORDEL E, LETER G. Sperm chromatin damage impairs human fertility. The Danish First Pregnancy Planner Study Team. Fertil Steril 2000; 73: 43-50.

32. Bungum M, Humaidan P, Spano M, Jepson K, Bungum $\mathrm{L}$, Ginercman A. The predictive value of sperm chromatin structure assay (SCSA) parameters for the outcome of intrauterine insemination, IVF and ICSI. Hum Reprod 2004; 19: 1401-8.

33. Agarwal A, Aluamaneni SS. The effect of sperm DNA damage on assisted reproduction outcomes. A review. Minerva Ginecol 2004; 56: 235-45.

34. Gandini L, Lombardo F, Paol D, Caruso F, Eleuteri P, LETER G ET AL. Full-term pregnancies achieved with ICSI despite high levels of sperm chromatin damage. Hum Reprod 2004; 19: 1409-17. 